\title{
Des territoires Celtiques aux cités Romaines en Gaule septentrionale
}

\author{
Xavier Deru et Rémi Auvertin
}

Par les listes de ciuitates gauloises septentrionale que nous livrent Pline ou Ptolémée, ou les référence à des ciuitates, nationes ou pagi mentionnés sur des inscriptions, apparaît une ambiguïté : la plupart des noms qui qualifient ces territoires sont des noms ethniques, déjà présents dans les Commentaires de César, et témoignant ainsi d'une continuité territoriale entre la fin de l'Âge du Fer et le Haut Empire. C'est pourquoi certains chercheurs prennent l'organisation territoriale romaine comme fond géographique à leur enquête sur la Gaule chevelue1. St. Fichtl plaide lui-même pour une continuité forte : « À l'arrivée de César en Gaule, les différents peuples étaient organisés en ciuitates », ciuitas « dont une notion territoriale existe bel et bien », dès l'Âge du Fer². Le fait que César localise les peuples les uns par rapport aux autres ou en prenant des éléments naturels (rivières, forêts, etc.) et que cette localisation sommaire se comprend par rapport aux ciuitates du Haut Empire, plaide également en ce sens. Au contraire, Strabon indique que les « divisions administratives établies par les chefs d'état, varient selon l'opportunité ».

Faute de sources décisives, nous écarterons l'hypothèse d'une continuité stricte des territoires gaulois aux ciuitates romaines et partirons d'un postulat où les communautés indigènes ne s'identifient pas à un territoire, mais correspondent à des clans et des lignages, ayant une langue, des pratiques sociales, religieuses, productives et de consommations communes. Les territoires que ces communautés occupent ne sont a priori ni limitrophes, ni distincts, ni distants.

Si l'on rejette la posture d'une continuité, notre raisonnement reposera, lui aussi, sur plusieurs présupposés que l'on justifiera, sans pouvoir les prouver. Ainsi notre enquête reposera premièrement sur l'hypothèse de la transmission des territoires romains aux territoires ecclésiastiques médiévaux.

1 S. Hornung, Siedlung und Bevölkerung in Ostgallien zwischen gallischem Krieg und der Festigung der Römische Herrschaft (Francfort 2016).

2 S. Fichtl, Les peuples gaulois. III ${ }^{\mathrm{e}} \mathrm{I}^{\text {er }}$ siècle avant J.-C. (Paris 2012), 5 ; 20. 
Deuxièmement, on considèrera comme valide une série d'attributions de monnaies de la fin de l'Âge du Fer à des peuples particuliers et ensuite, en troisième lieu, sur la concordance entre les aires de circulation de ces monnaies et des territoires occupés par ces peuples. L'acceptation de ces trois présupposés nous permettra alors de comparer les territoires de la fin de l'Âge du Fer et ceux de la période romaine. Cette démarche fera apparaître l'impact faible ou fort, causé par Rome sur l'identité territoriale de ces peuples.

\section{1 \\ Les présupposés}

\subsection{Les ciuitates romaines et les diocèses médiévaux}

La première étape de notre démarche comparative entre les périodes laténienne et romaine passe par la restitution des limites des cités romaines de Gaule Belgique et de Germanie. En l'absence d'une documentation antique directe, le tracé de ces limites repose traditionnellement soit sur l'application de modèles spatiaux (principalement celui des polygones de Thiessen ${ }^{3}$ ), soit sur une démarche régressive. C'est cette dernière que nous avons retenue, s'appuyant sur le postulat d'une continuité territoriale entre cités antiques et diocèses médiévaux ${ }^{4}$. Ce postulat de continuité repose pour partie sur les actes conciliaires tardo-antiques: le concile de Nicée en 325 pose le cadre provincial comme référence pour l'installation des évêchés (Nicaen. Canon. 4); le concile de Sardique en 343 permet la formation de nouveaux diocèses, centrés sur les grandes villes de l'Empire (Conc. Sard. a. 343, can. 2.1.2); enfin, les conciles mérovingiens et carolingiens affirment à plusieurs reprises la stabilité des limites diocésaines face aux découpages territoriaux promulgués par les rois ou aux tensions entre évêques (par exemple Conc. Epaon. a. 517, can. 9 ;

3 La méthode est habituelle dans la recherche néerlandaise, de J.H.F. Bloemers, «Engels drop. Een poging tot ontleding van het romanisatieproces in Nederland, Westerheem 29 (1980), 152-173 à N. Roymans, From the Sword to the Plough. Three Studies on the Earliest Romanisation of Northern Gaul (Amsterdam 1996). Pour une application récente, F. Vermeulen et D. Mlekuz, <Roman towns and the space between them: a view from northern Picenum >, dans F. Vermeulen et al. (éds.), Urban Landscape Survey in Italy and the Mediterranean (Oxford 2012), 207-222.

4 L'approche régressive a pu être appliquée, ces dernières années, aux cités des Bituriges, des Éduens, des Arvernes, des Trévires, des Atrébates ou des Nerviens. Voir notamment la discussion de la méthode au sujet de la cité des Bituriges: F. Dumasy, <Les limites de la cité des Bituriges >, dans C. Batardy, O. Buchsenschutz et Fr. Dumasy (éds.), Le Berry antique: Atlas 2000 (Paris 2001), 21-23; C. Gandini et al. (éds.), < Limites et marqueurs du territoire. L'approche de la ciuitas des Bituriges Cubes >, Caesarodunum 45-46 (2011-2012), 275-309. 
Conc. Lugd. a. 518, can. 3 ; Conc. Aruern. a. 535, can. 10). Ce principe de continuité, régulièrement soutenu ou mis en doute par la recherche, constitue de fait le seul levier permettant la cartographie, même approximative, des cités antiques et constitue l'axiome soutenant la majorité des cartes employées par les archéologues et historiens de la Gaule romaine.

Alors que la cartographie des cités romaines s'est longtemps appuyée sur les cartes de diocèse établies à partir $\mathrm{du} \mathrm{XVI}^{\mathrm{e}} \mathrm{s}$. et sur l'histoire diocésaine, notre propre démarche repose sur la confrontation de trois niveaux de données au sein d'un système d'informations géographique intitulé «Atlas des provinces romaines de Belgique et de Germanie ». Le diocèse médiéval peut être précisément cartographié à partir du XIII ${ }^{\mathrm{e}}$ s. et peut être comparé aux rares données antiques disponibles (routes, miliaires, sanctuaires, etc.). Considérant la rupture chronologique entre la période romaine et le Moyen Âge central et finissant, nous avons également ajouté, à la suite de B. et de R. Delmaire, une étape intermédiaire, celle du diocèse mérovingien ${ }^{5}$. Ce territoire, plus proche de la cité antique, n'est pas directement cartographiable mais peut être appréhendé à l'aide d'une autre catégorie territoriale mérovingienne, le pagus.

La confrontation des trois jeux de données, antique, mérovingien et médiéval, au sein du SIG, permet dès lors de proposer une restitution hypothétique des limites de cités antiques, fondée sur les plus anciennes sources disponibles (fig. 10.1 et 10.2). Par commodité et par habitude cartographique, par la suite, nous figurons ces limites sous forme de traits continus, bien que nous soyons conscients de l'existence de zones grises.

Largumentation complète de la démarche régressive et la discussion des résultats pour la restitution des territoires antiques et alto-médiévaux, objets d'une communication au cours du colloque «Far West» tenu à Caen en 2018, seront abordés dans un article indépendant ${ }^{6}$.

5 R. Delmaire et B. Delmaire, < Les limites de la cité des Atrébates (nouvelle approche d'un vieux problème) >, Revue du Nord 288 (1990), 697-735. Une application de cette étape intermédiaire est proposée dans X. Deru, <Cadres géographiques du territoire des Nerviens >, Revue du Nord 383 (2009), 179-201.

6 R. Auvertin et X. Deru, < Déterminer les territoires de cités au sortir de la guerre des Gaules : méthodologie d'une enquête entre Seine et Rhin >, dans Far West: la Normandie antique et les marges nord-ouest de l'Empire romain ( fin du I rer siècle av. J.-C.-VI siècle ap. J.-C.), colloque tenu à l'Université Caen Normandie et au musée départemental de Vieux-la-Romaine du 24 au 26 octobre 2018 (a venir). 


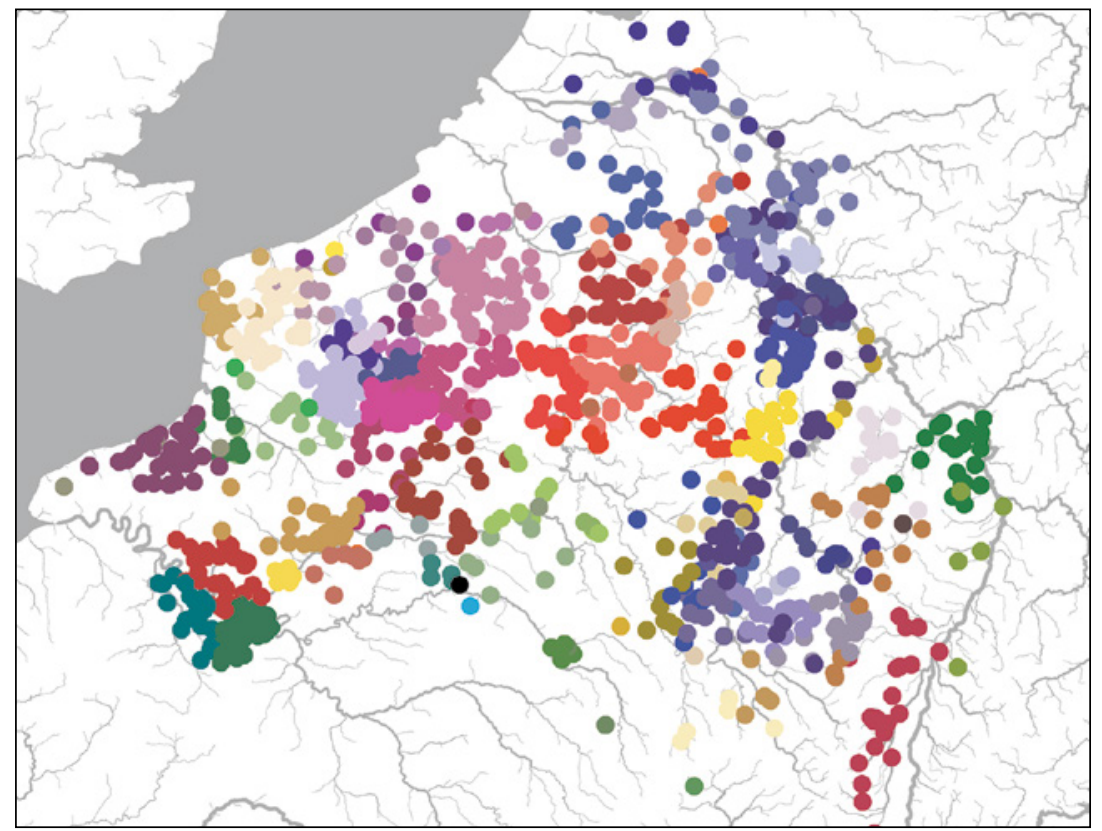

FIGURE 10.1 Localisation des lieux in pago, datant du Haut Moyen Âge (C) $\mathrm{ABG}$ )

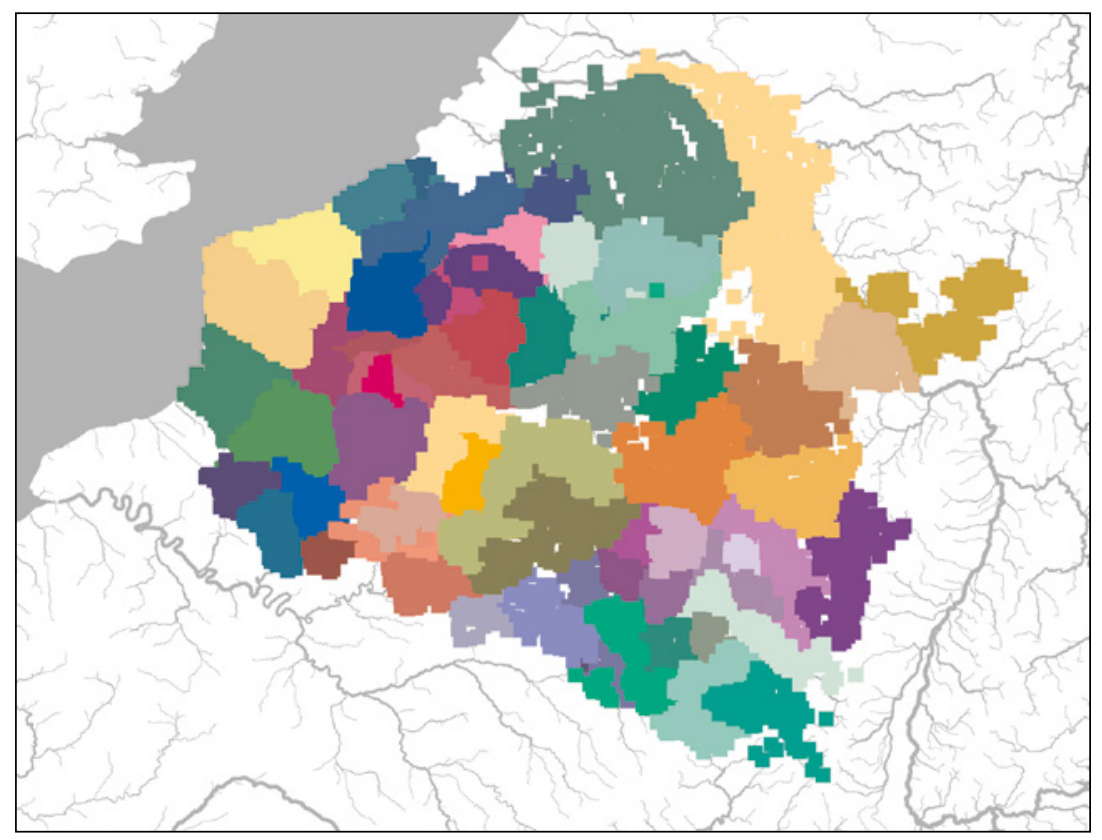

FIGURE 10.2 Localisation des paroisses médiévales par archidiaconé et diocèse ( (C) ABG) 


\subsection{Les monnaies et les territoires gaulois}

Comme nous l'avons indiqué, notre démarche repose sur la similarité entre les aires de circulation monétaire et les emprises territoriales des peuples. Pour certains archéologues, les monnaies gauloises sont avant tout un moyen d'échange et un témoignage de la prospérité économique 7 . A l'Âge du Fer, l'économie restait cependant peu monétisée, mais quoi qu'il en soit, son rôle dans les échanges, s'il existait, ne porte pas préjudice à notre démarche, mais pourra être souligné ponctuellement.

Les monnaies gauloises ont fait l'objet de classification typologique à une date ancienne, mais pour la Gaule septentrionale, correspondant à la Belgique, la typologie de $\mathrm{S}$. Scheers $(\mathrm{Sch}$.) constitue la référence-clé8, malgré les mises à jour de G. Depeyrot ou de L.P. Delestrée et M. Tache (DT) ${ }^{9}$. L'attribution des types monétaires à un peuple nécessite une bonne représentativité de l'effectif et une répartition cohérente avec une ciuitas romaine. Il convient d'admettre que notre raisonnement est ici circulaire; nous verrons qu'il s'agit seulement d'une étape qui ne nuit pas à l'enquête, ni à l'objectif poursuivi.

Laire de circulation des monnaies peut cependant dépasser le territoire du peuple ou du prince qui les a émises. Des critiques justifiées sur la validité de la répartition des monnaies comme témoignage d'un territoire ethnique ont été formulées notamment par K. Gruel ${ }^{10}$. En réalité, comme l'a mis en perspective St. Martin, la production et la circulation monétaire dans le Centre-Est de la Gaule diffèrent de celles de la Belgique, qui se caractérisent par son morcellement ${ }^{11}$.

On ignore évidemment si des lois fixent la circulation monétaire, comme dans certaines cités grecques, où règne l'exclusivité de l'usage de la monnaie locale ${ }^{12}$. Toutefois, une règle générale veut que les monnaies de faible valeur intrinsèque, en bronze ou en potin, gagnent en valeur fiduciaire, mais

$7 \quad$ Hornung 2016, op. cit. (n. 1), 263.

8 S. Scheers, Traité de numismatique celtique. II. La Gaule Belgique (Paris 1977).

9 G. Depeyrot, Numéraire celtique. VI. De la Manche au Soissonnais (Wetteren 2005);

G. Depeyrot, Numéraire celtique. VII. La Gaule orientale (Wetteren 2005); ‘Chronique numismatique >, Revue du Nord - Archéologie, jusqu'à aujourd'hui ; Carte archéologique de la Gaule avec une liste : https://www.aibl.fr/publications/collections/carte-archeologique -de-la-gaule/?lang=fr (dernier accès : 23 mars 2021); L.P. Delestrée et M. Tache, Nouvel atlas des monnaies gauloise. De la Seine au Rhin (Saint-Germain-en-Laye 2002).

10 K. Gruel, < Monnaies et territoires > dans D. Garcia et F. Verdin (éds.), Territoires celtiques. Actes du XXIV e colloque de l'AFEAF, Martigues juin 2000 (Paris 2002), 205-212.

11 S. Martin, Du statère au sesterce : monnaie et romanisation dans la Gaule du Nord et de l'est, III ${ }^{e}$ a.C.- ${ }^{\text {er }}$ s. p.C. (Bordeaux 2015).

12 C. Lauwers, Production et usages monétaires en Gaule du Nord (III ${ }^{e}$ s. avantJ.-C.-IX ${ }^{e}$ s. après J.-C.) (Bruxelles 2018). 


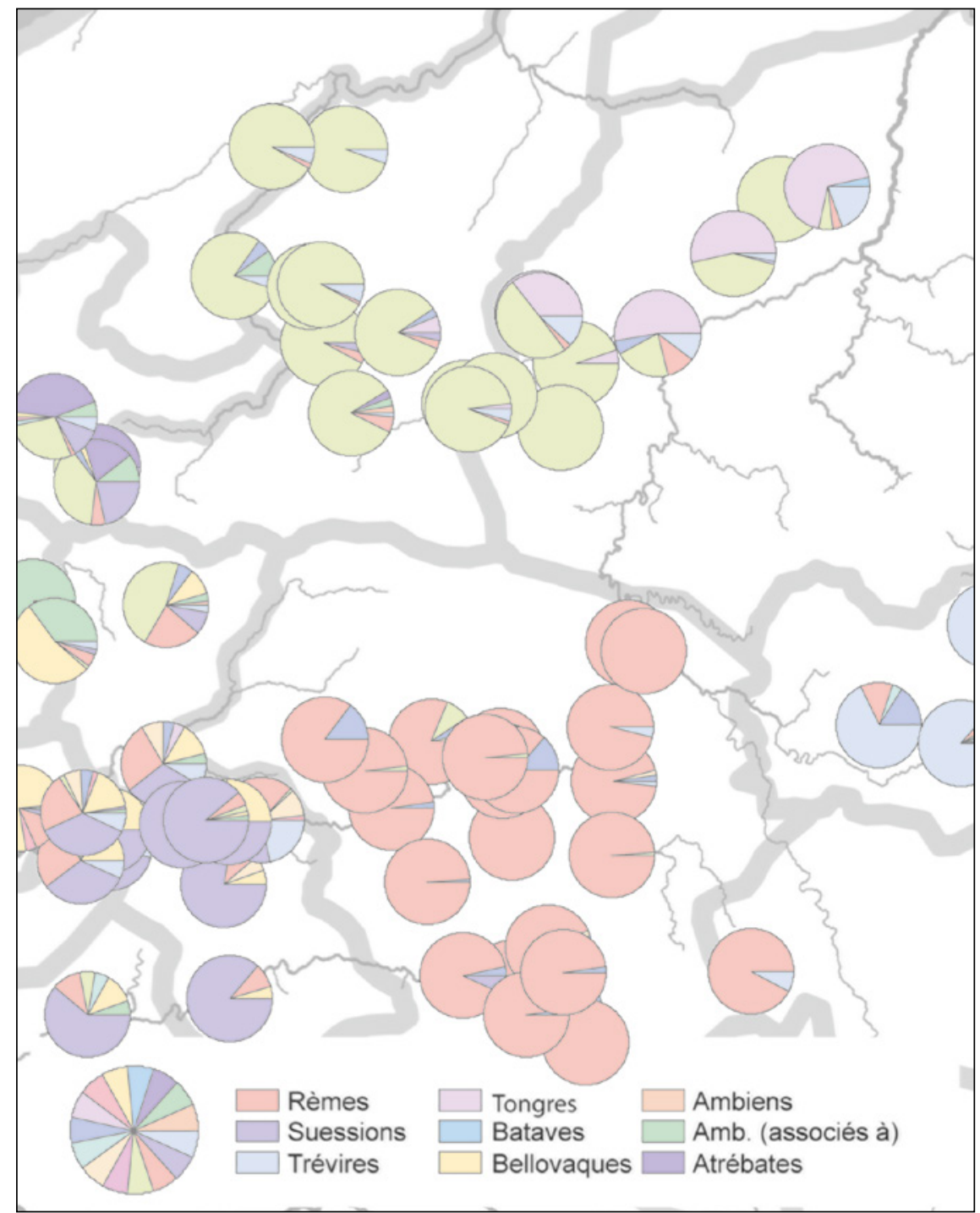

FIGURE 10.3 Effectifs monétaires par peuple pour les sites les mieux dotés (C) $\mathrm{ABG}$ )

uniquement dans le territoire du pouvoir émetteur. Pour cette raison, nous privilégierons celles-ci au détriment de l'or et de l'argent. De plus, la division des peuples devait entraver les échanges entre leur territoire. Sur la première carte est illustré le centre de la Belgique avec les sites ayant livré plus de vingt-quatre monnaies (fig. 10.3). On observe la nette majorité des monnaies soit rèmes, soit suessionnes, soit nerviennes, etc. sur des sites regroupés dans des régions 
circonscrites, ce qui témoigne de la faible circulation des monnaies d'un peuple à l'autre.

Les aires de circulation monétaires seront donc principalement appréhendées par les monnaies de faibles valeurs, nombreuses et bien réparties; des frappes d'une même période, de la fin de l'indépendance ou postérieure à la Guerre sont associées. Ces monnaies sont répertoriées au sein notre sIG dans une table typologique et une autre d'inventaire. 50 o 33 monnaies sont ainsi répertoriées, cet inventaire a débuté par le dépouillement critique des volumes de G. Depeyrot et d'autres volumes synthétiques ${ }^{13}$; nous citerons ensuite quelques publications-clés liées à des peuples particuliers. Pour chaque peuple, nous en avons projeté les trouvailles individuelles et fusionné celles-ci par site ; les effectifs sont repris dans tableau 10.1; ensuite, nous avons calculé l'aire de densité des lieux de découverte selon la méthode des noyaux (spatial Analyst, ArcGis) selon l'optimisation statistique du logiciel, enfin ces aires de densité sont illustrées par des isolignes. Toutes les cartes sont à la même échelle et présentent en filigranes l'emprise des cités et le réseau hydrographique. Sur chacune d'elles, une aire de densité principale apparaît sur une zone géographique cohérente, mais il est évident que des aires résiduelles demeurent à cause de sites où le monnayage est très volumineux, mais qui, à cause de cela, compte toujours quelques monnaies exogènes.

\section{Les aires de circulations monétaires}

Un premier cas illustre la validité de notre démarche, puisque les Rèmes ont frappé des monnaies sur lesquels l'ethnique est donné : REMO. Par cela, trois types sont assurément attribuées à ce peuple ${ }^{14}$. La monnaie REMO REMO (Sch. 146) est le type le plus abondant et celui qui présente l'aire de distribution la plus vaste et la plus dense, notamment parce que sa circulation se poursuit au début de la période romaine (fig. 10.4A). L'aire de circulation s'inscrit dans le territoire de la cité romaine, en débordant dans la vallée de l'Aisne, soit pour des raisons économiques, soit à cause des relations de clientèles entre les Rèmes et les Suessions.

13 J.L. Dengis, Trouvailles et trésors monétaires en Belgique. VI. Les monnaies gauloises (Wetteren 2010); Depeyrot 2005, op. cit. (n. 9). 〈Chronique numismatique >, Revue du Nord - Archéologie, jusqu'à aujourd'hui, op. cit. (n. 9); Carte archéologique de la Gaule, op. cit. (n. 9) pour les trouvailles monétaires.

14 J.-M. Doyen, Structure et dynamique des flux monétaires chez les Rèmes: modélisation d'un traceur socio-économique entre 275/250 av. et 68 apr. J.-C., Habilitation à Diriger les Recherches, Université de Nice-Sophia Antipolis, 11 décembre 2014. 

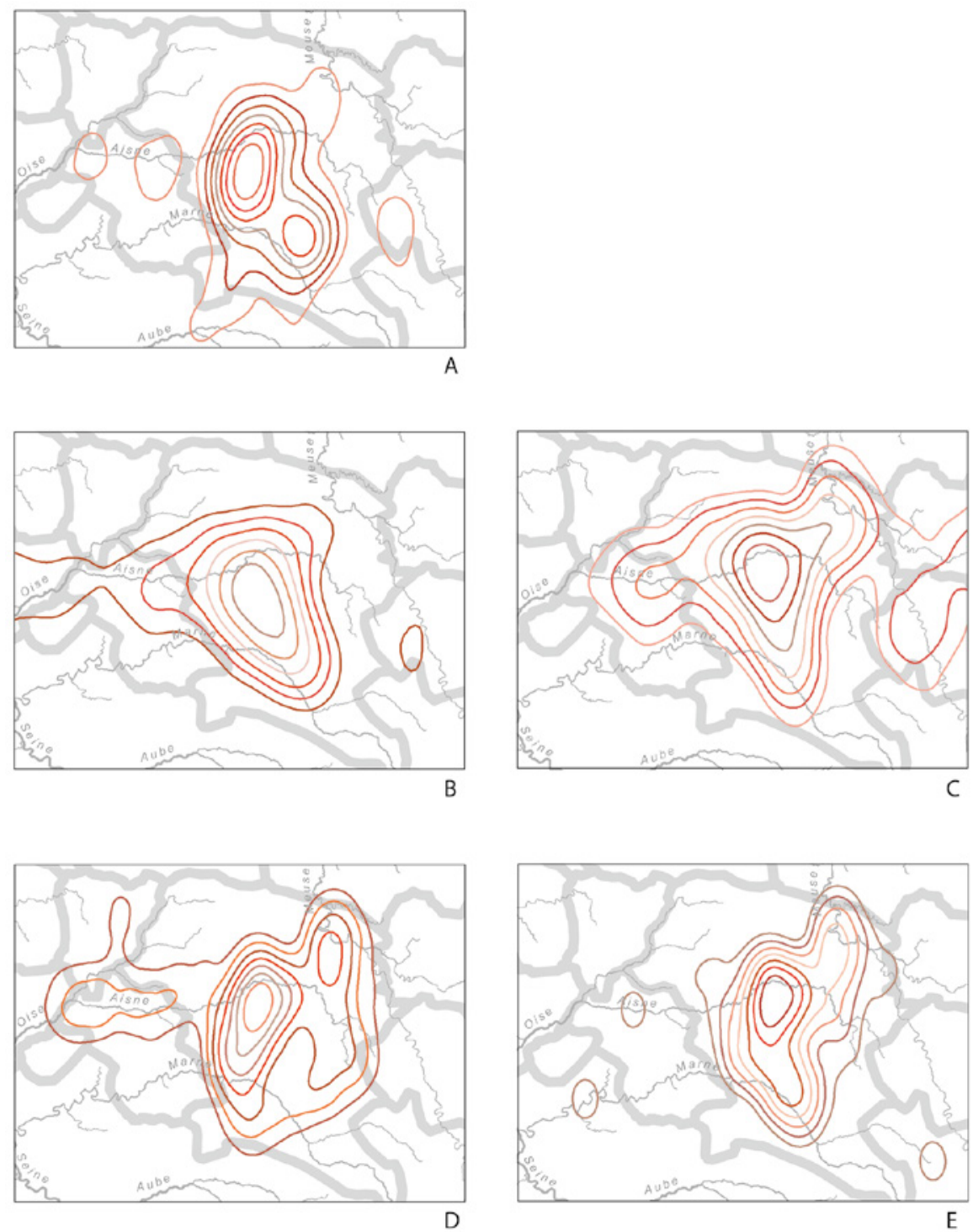

FIGURE 10.4 Densité de la répartition des monnaies des Rèmes (A. Sch. 146 ; B. Sch. 191 ; C. Sch. 194 ; D. Sch. 147 ; E. Sch. 151)

(C) ABG)

Si l'on prend les monnaies rèmes les plus communes, antérieures à la Guerre des Gaules (Sch. 191/192 et Sch. 194/195; fig. 10.4B/C) ou postérieures à celle-ci (Sch. 147 et Sch. 151 ; fig. 10.4D/E), la distribution est identique dans les lignes générales et couvrent les deux tiers de la cité romaine. Il convient de noter que le nord-ouest et le sud-est de la cité ne sont pas recouverts, point sur lequel nous reviendrons plus tard. 

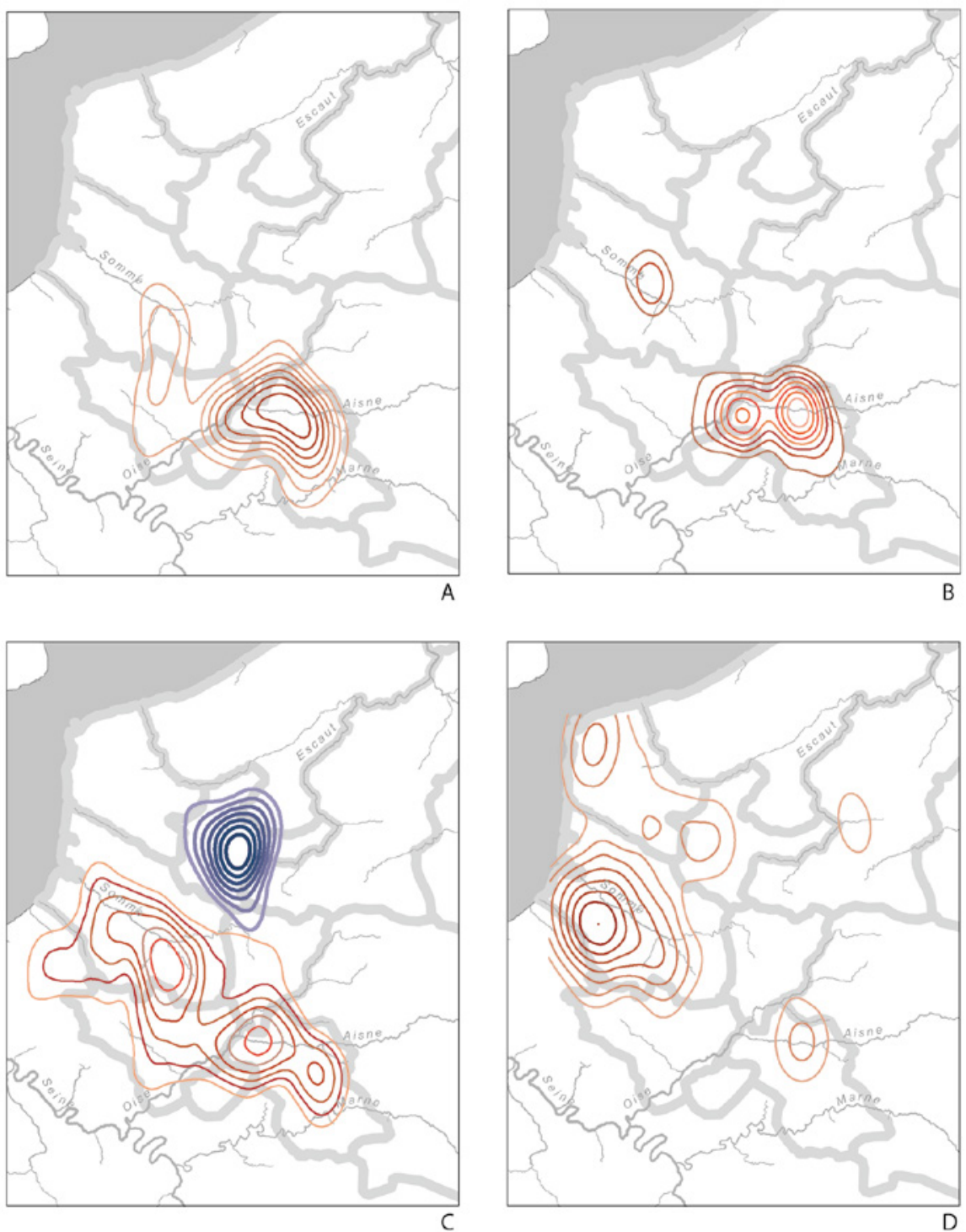

FIGURE 10.5 Densité de la répartition des monnaies des Suessions (A. Sch. 27A ; B. Sch. 196), des Bellovaques et des Atrébates au nord (C) et des Ambiens (D) (C) $\mathrm{ABG}$ )

Des Suessions, trois types ont été sélectionnés à partir du nombre d'occurrences. Les monnaies de Cricironus (Sch. 27 ; fig. 10.5A) et celles de Diviciacos (types Sch. 155/157, non ill.) sont antérieures à la Guerre, les types Sch. 196/197 sont, quant à eux, postérieures (fig. 10.5B). L'aire majeure des découvertes se circonscrit à l'Aisne ; elle ne s'étend pas au territoire rème, ce qui va à l'encontre des relations de clientèle, alors que son débordement en aval, sur le territoire 
des Bellovaques s'explique par le sens du courant. Un fait particulier est l'absence de ces monnaies au sud de la Marne que César avait d'ailleurs donné comme limite à la Belgique.

Les monnayages en bronze des Bellovaques (Sch. 120-130/135/163; fig. 10.5C) ont été rassemblés sur une seule carte. A moins qu'il y ait des erreurs d'attribution, ce que nous ne croyons pas, car pris séparément, chaque type présente la même répartition, les monnayages s'étendent largement sur le territoire des Suessions et celui des Ambiens.

De même, nous avons sélectionné plusieurs types monétaires en bronze qui appartiennent vraisemblablement aux Ambiens et qui datent d'avant la Guerre : Sch. 24 cl.7, 8od ; DT245/246/384/389/395/415/468/472-475 (fig. 10.5D). Ces monnaies recouvrent la ciuitas romaine, mais s'étendent aussi vers le nord, sur le territoire des Morins, dans l'arrière-pays de la Manche.

Plus à l'est, les Atrébates ont une série monétaire intéressante ${ }^{15}$. En particulier, les monnaies au nom de Commios (Sch. 45/47), leur roi, tantôt allié, tantôt ennemi de César, se localisent sur le territoire de la cité et plus au nord (non illustré). Toutes les monnaies antérieures à la Guerre des Gaules, qu'elles soient en or, en argent et en bronze ont été réunies pour des raisons statistiques: Sch. 11, 45-47 et 136 (fig. 10.5C). Elles montrent aussi la densité la plus forte sur l'emprise de la cité romaine.

Plus que les Atrébates, les Nerviens ont été fortement affectés lors de la Guerre. C'est pour ce peuple que notre démarche a été pour la première fois appliquée ${ }^{16}$. La distribution des monnaies en potin et en bronze (Sch. 145 and 190 ; fig. 10.6A/B) semble se concentrer dans le Hainaut, alors que le Cambrésis au sud et le plateau brabançon au nord en sont dépourvues. La limite entre le Hainaut et le Cambrésis est marquée par la Selle, un affluent de l'Escaut identifié au Sabis, rivière où s'est tenue la terrible bataille de 57 avant J.-C. Au contraire, si les monnaies dépassent les limites de la cité, elles se distribuent sur un axe est-ouest, dans la partie méridionale de la cité des Ménapiens et chez les Atuatuques, jusqu’à la Meuse.

Comme les statères en or, les monnaies en bronze (Sch. 58, 217 and 165; fig. 10.7A) présentent deux cœurs, l'un sur le Waal, dans le territoire des Bataves, l'autre plus en amont sur la Meuse, chez les Eburons, les Atuatuques ou les Tongres selon la datation des monnaies. ${ }^{17}$

15 D. Gricourt, < Les monnayages tardifs des Atrébates, témoins de la destinée éphémère d'un royaume celto-romain >, dans J. van Heesch et I. Heeren (éds.), Coinage in the Iron Age. Essays in honour of Simone Scheers (Londres 2009), 123-153.

16 Deru 2009, op. cit. (n. 5).

17 S. Scheers, < Frappe et circulation monétaire sur le territoire de la future Civitas Tungrorum >, RBelgNum 142 (1996), 5-51 ; N. Roymans, G. Creemers et S. Scheers (éds.), Late Iron Age gold 


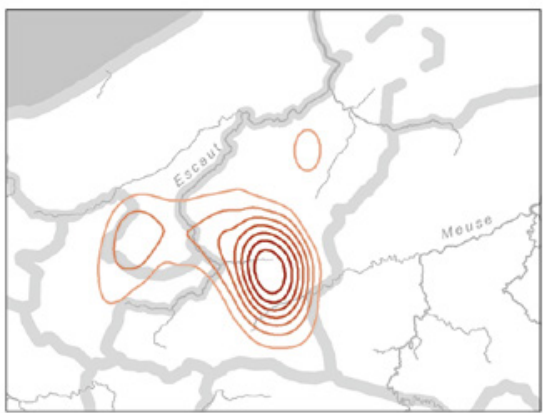

A

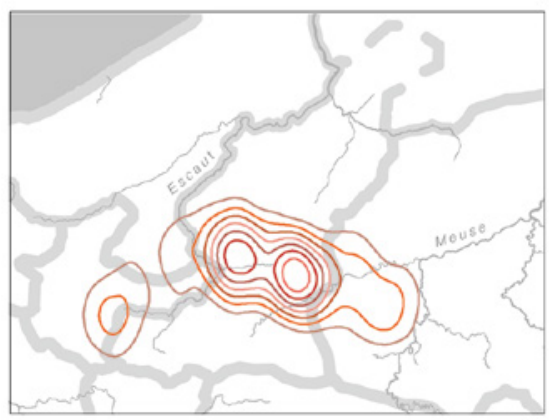

B

FIGURE 10.6 Densité de la répartition des monnaies des Nerviens (A. Sch. $145 ;$ B. 190) (C) $\mathrm{ABG}$ )

Les monnayages des Trévires bénéficient du travail de Loscheider ${ }^{18}$. Les monnaies en bronze et en potin du premier horizon (Sch. 199-201; fig. 10.7B) se répartissent sur l'ensemble de la cité romaine et au-delà jusqu'au Rhin. Les monnaies en or et en argent montent également vers le nord, au travers des Ardennes, ce qui correspond au texte de César qui indique d'une part, que les Trévires sont voisins du Rhin et d'autre part, que les Condruses et les Pémanes vivent sous leur protection ${ }^{19}$. A la différence des monnaies anciennes, les monnaies postérieures à la Guerre (Sch. $30 \mathrm{~A}$ et 162 ; fig. $10.7 \mathrm{C} / \mathrm{D})$ montrent une réduction de leur circulation, limitée à l'emprise de la ciuitas. Cette rétraction peut s'expliquer par la militarisation du Rhin dans la seconde moitié du Ier s. avant J.-C.

Pour terminer ce tour de la Belgique, nous pouvons remonter le cours de la Moselle et aborder le massif vosgien. Ce territoire devait être occupé par les Médiomatriques et les Leuques, peuples pratiquement absents des

hoards from the low countries and the Caesarian conquest of Northern Gaul (Amsterdam $2012)$; N. Roymans et J. Aarts, < Coin use in a dynamic frontier region. Late Iron Age coinages in the Lower Rhine Area >, Journal of Archaeology in the Low Countries 1 (2009), 5-26. R. Loscheider, «Untersuchungen zum spätlatènezeitlichen Münzwesen des Trevererlandes >, AMosel 3 (1998), 63-225. Complété par F. Reinert, < La numismatique celtique au Luxembourg. Une réactualisation après 30 ans de fouilles et prospections archéologiques >, dans J. van Heesch et I. Heeren (éds.), Coinage in the Iron Age : Essays in Honour of Simone Scheers (Londres 2009), 337-361. D. Wigg, Koblenz: Der Martberg bei Pommern (Ehem. Cochem) (Mayence 2005); D. Wigg-Wolf, < The coin finds from the Donnersberg, Rheinland-Pfalz, Germany >, dans Heesch et Heeren 20o9, op. cit. (n. 18), 399-419. D. Krausse, Eisenzeitlicher Kulturwandel und Romanisierung im Mosel-Eifel-Raum: Die keltischrömische Siedlung von Wallendorf und ihr archäologisches Umfeld (Mayence 2006). 

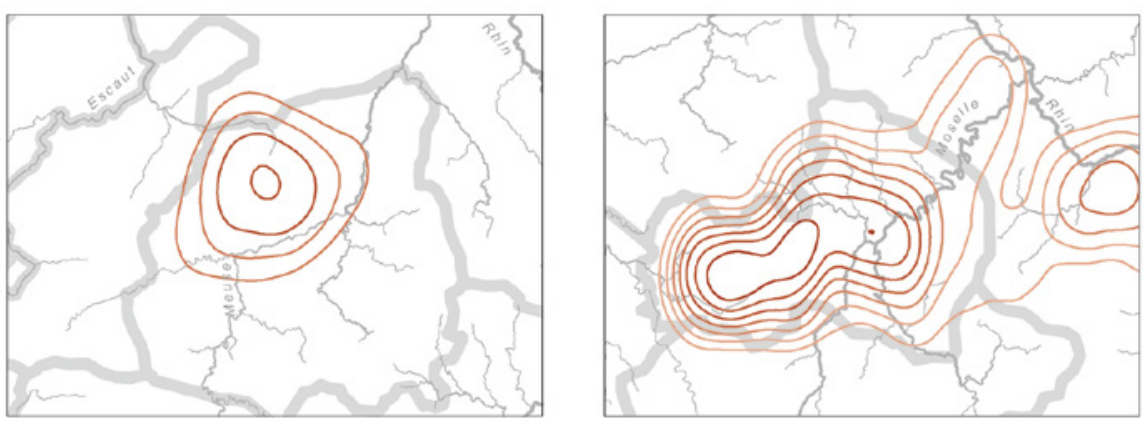

A
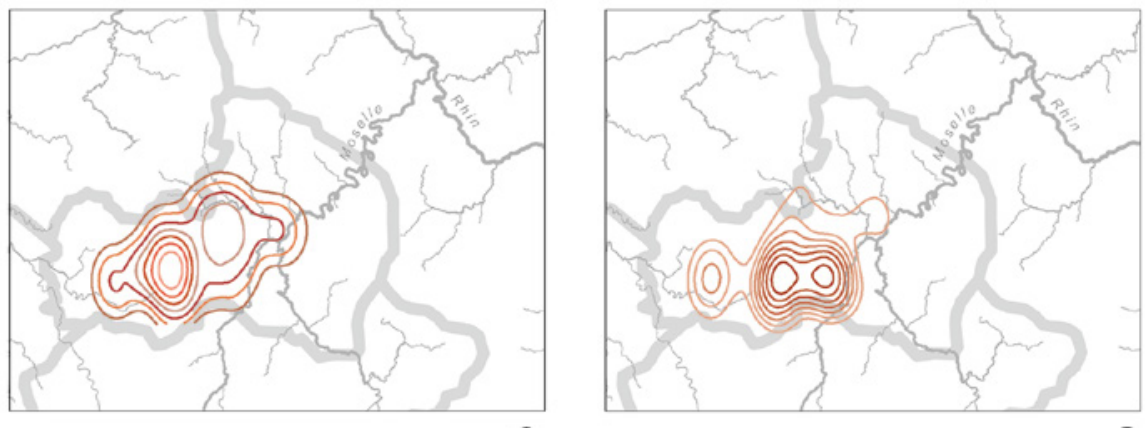

C

D

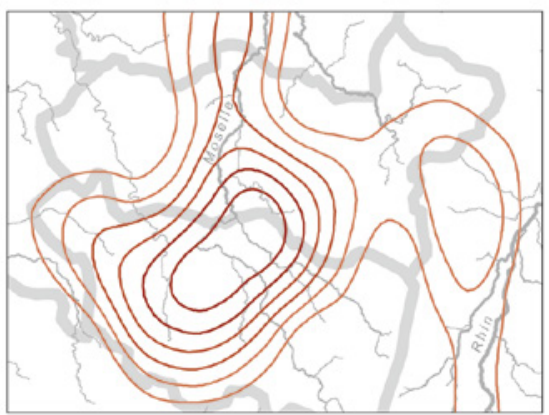

$\mathrm{E}$

FIGURE 10.7 Densité de la répartition des monnaies des Eburons/Tongres (A. Sch. 58, 165 et 217), des Trévires (B. Sch. 199 ; C. Sch. 162 ; D. Sch. 3०A) et des Médiomatriques/ Leuques (E. Sch. 186)

( (C) $\mathrm{ABG)}$

commentaires de César et qu'il est donc difficile de caractériser ${ }^{20}$. Comme Cl. Féliu ${ }^{21}$, nous pouvons utiliser le potin « au sanglier» (Sch. 186; fig. 10.7E),

$20 \quad$ Hornung 2016 , op. cit. (n. 1), 375-428.

21 C. Féliu, « Monnaies et frontières dans la vallée du Rhin supérieur à La Tène finale `, dans M. Schönfelder et S. Sievers (éds.), LẤge du Fer entre la Champagne et la vallée du Rhin. 
une monnaie antérieure à la Guerre, comme indicateur du territoire de ces peuples. La circulation couvre un territoire cohérent qui s'étend ensuite vers le nord, suivant le cours de la Moselle. On retrouve également cette monnaie chez les Rèmes, peut-être à cause d'une similarité typologique ${ }^{22}$.

Au préalable de la cartographie des peuples de la Belgique, il faut rappeler que faute de données statistiques fiables, les Morins, les Viromanduens ou les Silvanectes n'y ont pas été figurés, comme également, les peuples clients auxquels on n'a pas pu attribuer de monnaies. De plus, tous les peuples n'ont pas émis ou utilisé de monnaies.

Débarrassé d'une croyance en un continuité stricte qui permettrait de restituer les territoires de la fin de l'Âge du Fer à partir de la période romaine, mais en posant, il est vrai d'autres présupposés, nous pouvons proposer quelques éléments nouveaux pour la restitution de ces territoires ou pour comprendre comment l'impérialisme romain à réorganiser les territoires et les peuples conquis.

La carte synthétique tend à montrer que la totalité du territoire de l'Âge du Fer n'est pas partagée entre chaque peuple (fig. 10.8), que de vastes zones, comme la plaine maritime, l'Argonne ou le massif des Ardennes sont peu densément occupés et que les placer sous l'autorité d'un peuple particulier est sans doute inutile. La contiguïté des territoires et la présence de frontières ne sont donc pas, à l'Âge du Fer, des éléments nécessaires. Il est toutefois certain que dans les zones où l'occupation est plus forte, les territoires pouvaient avoir des populations mêlées, ou des frontières marquées dans le paysage, par tel relief, rivière ou zone humide.

Dans la partie occidentale, dans le Belgium, la création des ciuitates témoigne d'une grande continuité, en particulier pour les Atrébates, les Ambiens, les Bellovaques et les Suessions. Pour ces derniers, il semble que leur territoire ait été élargi vers le sud, au-delà de la Marne. Cet accroissement du territoire se voit également pour les Nerviens qui ont également obtenu le statut de cité libre, en vue peut-être d'y restaurer un développement démographique et économique. La cité fédérée des Rèmes est également plus large que l'aire de circulation monétaire, notamment au sud et au nord. Il convient de signaler que

Actes du XXXIVe colloque de l'AFEAF, Mai 2010 (Mayence 2012), 459-467 ; J.P. Lagadec, < La circulation monétaire celtique en Lorraine >, Archaeologia Mosellana 3 (1998), 9-6o ; E. Mériel, < La circulation monétaire celtique en Alsace `, RAE 51 (2001-2002), 215-25o. 


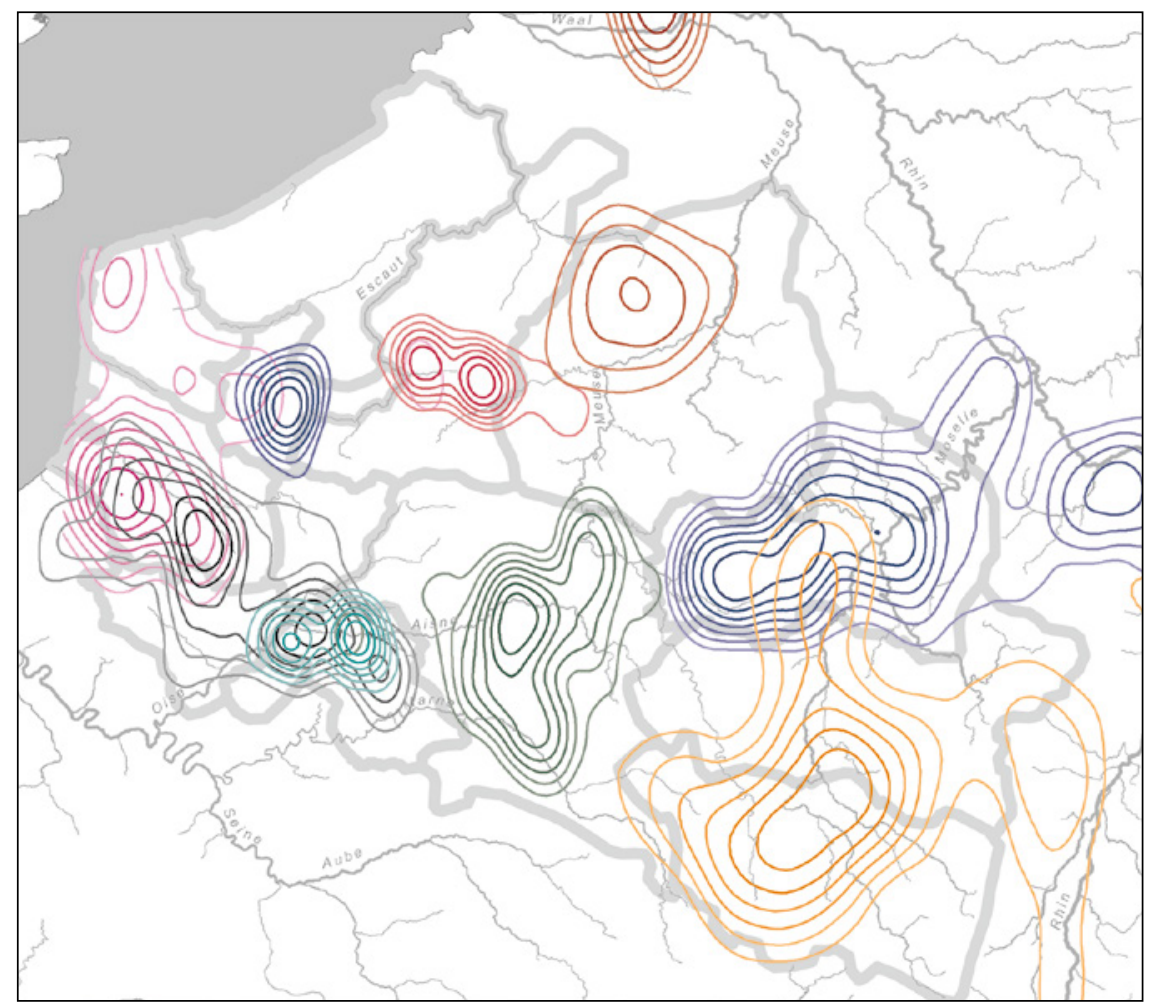

FIGURE 10.8 Synthèse des cartes 4-7

(C) $\mathrm{ABG}$ )

la partie sud, dans l'Antiquité tardive, constituera une cité propre, celle des Catuvelaunes et la partie nord, deviendra au Ve s. un diocèse particulier, celui de Laon. On pourrait considérer que pour des raisons opposées, Nerviens et Rèmes ont bénéficié de la politique romaine de reconstruction.

A l'est, les ruptures semblent plus significatives. Les Eburons ont été exterminés pendant la Guerre et bien que le chef-lieu, Tongres, s'appelle Atuatuca, les Atuatuques, eux-mêmes, n'ont pas nommé la cité. La ciuitas tungrorum intègre les territoires de ces peuples, mais également ceux au sud de la Meuse, le Condroz et la Famenne, qui étaient auparavant sous l'influence des Trévires. Nous sommes peut-être dans une situation proche, mais plus accentuée, de celle des Nerviens. Les Trévires apparaissent comme ceux ayant perdu le plus grand territoire : au nord, nous venons de l'indiquer, mais aussi à l'est, puisque la zone rhénane leur a été retirée au profit de l'autorité militaire. Malgré cette perte, la ciuitas est devenue l'une des plus puissante de Belgique, devenant une colonie, le siège du procurateur et un pôle économique clé sur la Moselle. 


\section{Conclusion}

Bien que Rome ait nommé les nouveaux territoires en se référant aux peuples indigènes, le degré de continuité doit être discuté. Rome a apporté une division globale et totale du territoire, avec des entités limitrophes, ayant un statut juridique spécifique. Le paysage est maintenant divisé et borné. Rome a recréé ces territoires au cas par cas, en jouant sur la continuité, la recomposition ou la négociation, en privilégiant certains au détriment d'autres. L'identité auparavant fondée sur la généalogie, la religion, la langue et la coutume, se définit maintenant par rapport à la cité ; c'est en son sein, comme entité de l'empire, que les communautés vont redéfinir leur culture.

TABLE 10.1 Effectif des monnaies par peuple et par type. Nombre total de monnaies, Nombre maximum de monnaie sur un site ; Nombre moyen de monnaies par sites ;

Nombre total de sites

\begin{tabular}{lllll}
\hline Peuples & Types (Scheers, DT) & Total & Max. & Moyen.
\end{tabular}

$\begin{array}{llrrrr}\text { Ambiens } & \text { 24 cl.7, 8od; } & 50 & 7 & 1,6 & 31 \\ & \text { DT245/.../472-475 } & & & & \\ \text { Bellovaque } & 120-130 / 135 / 163 & 1153 & 221 & 8,8 & 131 \\ \text { Rèmes } & 146 & 1144 & 187 & 6,6 & 173 \\ & 191 / 192 & 1860 & 195 & 5,5 & 337 \\ & 194 / 195 & 825 & 142 & 7,3 & 112 \\ & 147 & 652 & 134 & 9,8 & 66 \\ & 151 & 906 & 191 & 14,3 & 63 \\ \text { Suessions } & 27(\mathrm{AE}) & 1490 & 1084 & 19,8 & 75 \\ & 196 / 197 & 569 & 496 & 14,2 & 40 \\ \text { Atrébates } & 11,45-47,136 & 170 & 25 & 2,1 & 79 \\ \text { Nerviens } & 145 & 166 & 24 & 2,5 & 67 \\ & 190 & 1367 & 237 & 5,4 & 253 \\ \text { Trevires } & 199-201 & 798 & 451 & 8,7 & 91 \\ & 30 \mathrm{~A} \mathrm{cl.} \mathrm{2-5} & 2098 & 1540 & 33,3 & 63 \\ & 162 & 810 & 727 & 22,5 & 36 \\ \text { Eburons } & 58,165 \text { et } 217 & 1557 & 382 & 11 & 141 \\ \text { Médiom., } & 186 & 2903 & 561 & 13,6 & 212 \\ \text { Leuques } & & & & & \end{array}$

